F

\title{
Analyzing the behavior of bicyclists using a bicycle simulator with a coupled SUMO and DYNA4 simulated environment
}

\author{
Heather Kaths ${ }^{1}$, Andreas Keler ${ }^{1}$, Jakob Kaths ${ }^{2}$ and Fritz Busch ${ }^{1}$ \\ ${ }^{1}$ Technical University of Munich \\ ${ }^{2}$ TESIS GmbH \\ heather.kaths@tum.de, andreas.keler@tum.de, jakob.kaths@tesis.de, \\ fritz.busch@tum.de
}

\begin{abstract}
Operational behavior models are used in traffic simulations to represent the subconscious, short-term decisions made by road users to respond to other road users, the infrastructure and traffic control measures. Calibration and validation of these models can be achieved using observed trajectory data from real road users. For lane bound traffic, it is assumed that road users intend to follow a given lane with a certain desired speed across the intersection. Any deviation from this planned path is in response to other road users or the environment. It is difficult, however, to identify and separate the desired movement of more flexible road users that do not follow lane disciple, such as bicyclists, from movements made as a reaction to other road users or obstacles. This can lead to poor calibration of operational behavior models and unrealistic behavior in the simulation. Tactical behavior models recreate the conscious decisions made on a time horizon of seconds to minutes to cope with the immediate traffic situation. As such, tactical behavior models are responsible for selecting the planned path across an intersection.

Here, SUMO is coupled with the simulation software DYNA4 to create a simulated road environment for a bicycle simulator. Trajectories observed in reality are displayed as potential prescribed pathways across the simulated intersection. Participants in the simulator study are instructed to select and follow one of the prescribed pathways as closely as possible while responding naturally to other road users and obstacles in the environment. The resulting trajectory data is used to calibrate existing operational and tactical path finding behavior models for bicyclists at signalized intersection.
\end{abstract}




\section{Introduction}

As a mode of transportation, the bicycle is space efficient, non-polluting, quiet and promotes a healthy lifestyle. These features make bicycling an increasingly attractive solution to many of the problems associated with transportation in urban areas. However, urban planners and engineers face many obstacles in designing and building urban infrastructure that is safe and efficient for all modes of transport.

An important tool for testing and evaluating infrastructure design options and traffic control measures is microscopic traffic simulation. This tool makes it possible to simulate many scenarios and evaluate potential effects prior to real world implementation. The validity of such evaluations, however, is strongly dependent on the realism of the behavior models used within the simulation software. Modelling the behavior of bicyclists for microscopic traffic simulation is a particularly challenging task due to the flexibility and lack of lane discipline of these road users. Although traffic laws in many countries stipulate the movement of bicyclists at intersections, bicyclists tend to utilize available infrastructure (roadway, bicycle lanes and sidewalks) to cross an intersection using unexpected pathways (Amini, Twaddle, \& Leonhardt, 2016).

An approach for modelling the operational behavior of bicyclists at intersections is proposed by Twaddle (2017). This model is based on the premise that bicyclists aim to follow a tactically selected pathway across an intersection. They deviate from this planned pathway as a response to other road users, the traffic signal or other obstacles. The calibration and validation of this model was carried out using observed trajectory data. As it was not possible to observe the intended pathway of each bicyclist, the complete dataset of observed trajectories was used to estimate this pathway. This estimation introduced an error to the calibration of the model.

Similarly, a multinomial regression model for the path selection of left turning bicyclists was described by Twaddle (2017). Static and dynamic characteristics of the intersection are used to predict the pathway a bicycle will use to cross the intersection. The model was calibrated and validated using the same observed trajectory data. In this case, the observed data included all necessary information to estimate and evaluate the tactical behaviour model.

In this paper, a bicycle simulator experiment is presented, in which study participants are instructed to select and then follow a path across an intersection. The actual trajectories of the simulator study participants can be compared to the given pathways to understand the reactive behaviour of bicyclists and to calibrate and validate the operational behaviour model proposed by Twaddle (2017). The path selection of the bicyclists is used to estimate a multinomial regression model. The bicycle simu lator can be validated by comparing and contrasting the resulting models from the real world and the simulator study.

\section{Methodology}

An approach is developed and presented for connecting three environments; observations from an intersection in the real world, a SUMO traffic simulation and a DYNA4 simulation of the road environment. The proposed methodology allows for simulator study participants to bicycle across a simulated real intersection and interact with simulated road users. The road users in the simulated environment are driven by behavior models (SUMO traffic simulation). In this section, the bicycle simulator hardware is briefly described, followed by an explanation of the coupled simulated environment. The inclusion of pathways observed at the real world intersection is then presented. 


\subsection{Bicycle simulator}

The bicycle simulator at the Chair of Traffic Engineering and Control at the Technical University of Munich (TUM VT) was built with the purpose of researching and quantifying the behavior of bicyclists using a controlled test environment. The hardware and software components of the bicycle simulator are described in detail in the paper A bicycle simulator for experiencing microscopic traffic flow simulation in urban environments (Keler et al., 2018). The current simulator operates using the workflow depicted in Figure 1.

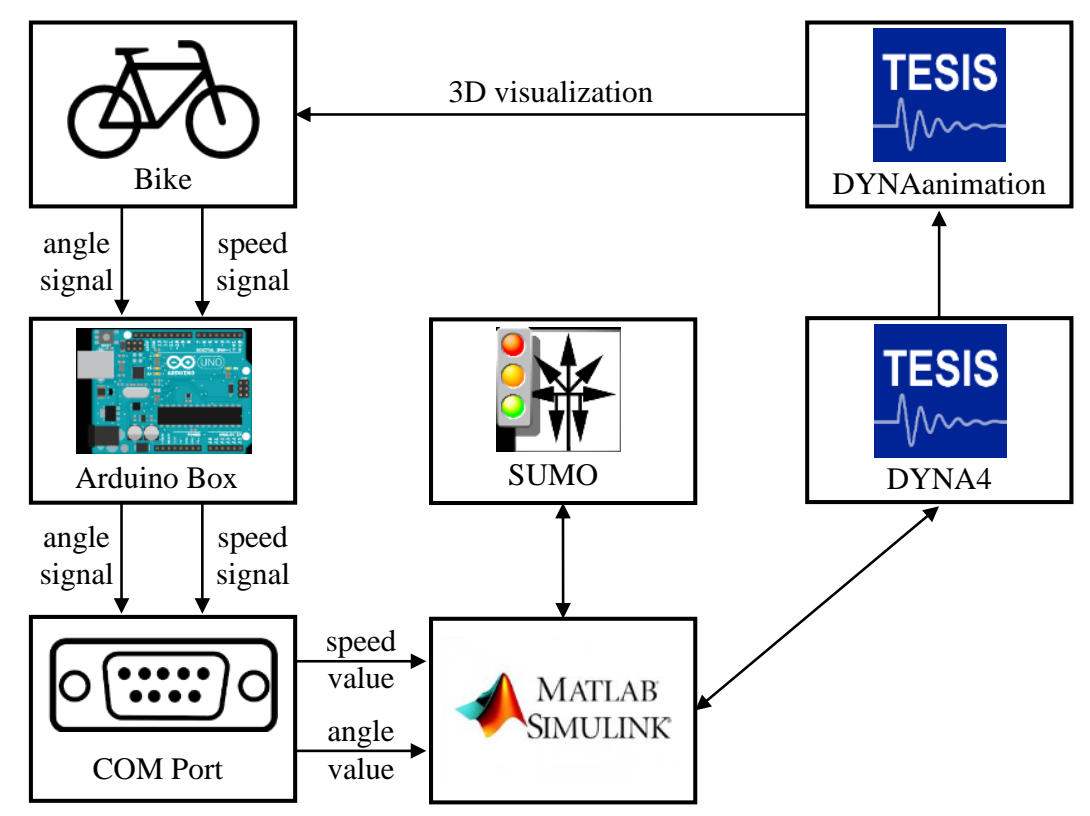

Figure 1: Workflow of the current bicycle simulator at TUM VT (Keler et al., 2018)

The direction of the front wheel is measured using a magnetic rotary encoder and the rotational speed of the rear wheel is read using an infrared distance measuring device. The raw speed and angle signals are processed using an Arduino box and passed to the computer using the COM port. MATLAB SIMULINK is used to manage and control the signal input from the COM port, the traffic simulation SUMO and the environment simulator DYNA4. A single-track model in DYNA4 converts the raw input from the sensors into a speed and direction of travel (velocity) of the bicycle, enabling the positioning and orientation of the bicyclist in the simulated environment. Surrounding traffic is created using SUMO and the movements of the other road users are controlled using native driver behavior models in SUMO. The simulated environment and other road users are visualized with DYNAanimation. The coupling of SUMO with DYNA4 (Kaths, Schott, \& Chucholowski, 2018) makes it possible to easily and quickly manipulate the characteristics of the surrounding traffic (e.g. traffic volume and composition) and add stochasticity to simulator experiments. However, the number of visualized road users is currently restricted in DYNA4. Either virtual reality glasses or a combination of monitors can be used depending on the preference and comfort of the participant. The bicycle simulator with the sensors for direction of the front wheel and the rotational speed of the rear wheel is shown in Figure 2. 


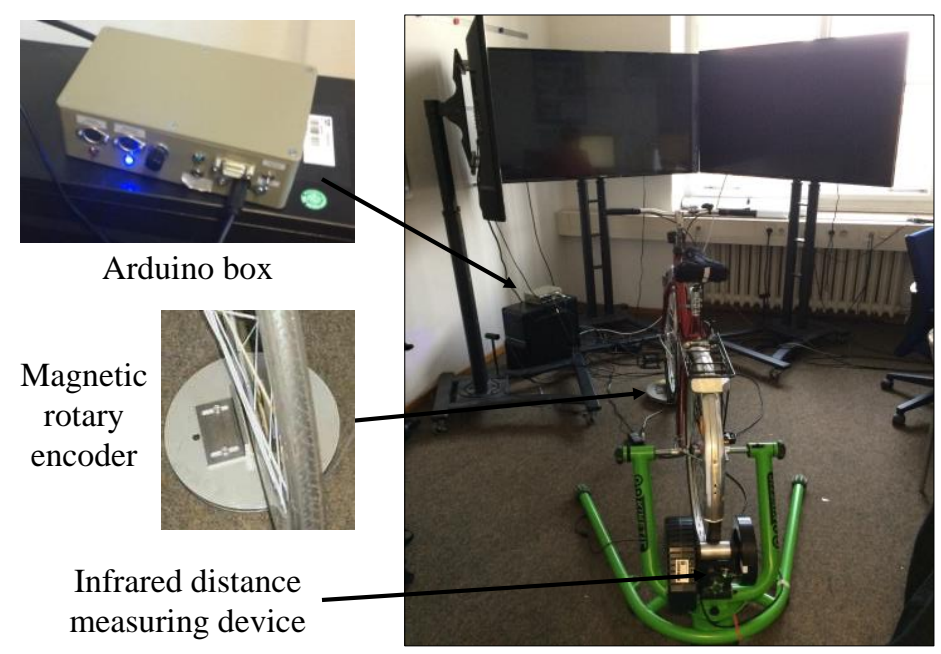

Figure 2: Bicy cle simulator setup at TUM VT

\subsection{Simulated environment}

The four arm, signalized intersection of Marsstraße and Seidlstraße in Munich, Germany is selected for the first application of this methodology. Video data was collected at this intersection in 2013 (camera view in Figure 3, left). A SUMO simulation network was built and vehicle and bicycle traffic was introduced based on traffic counts from the video data. The intersection environment was visualized using DYNA4. The pathways specified to guide the bicyclists across the intersection are shown in red, blue and green dots in Figure 3 (right).
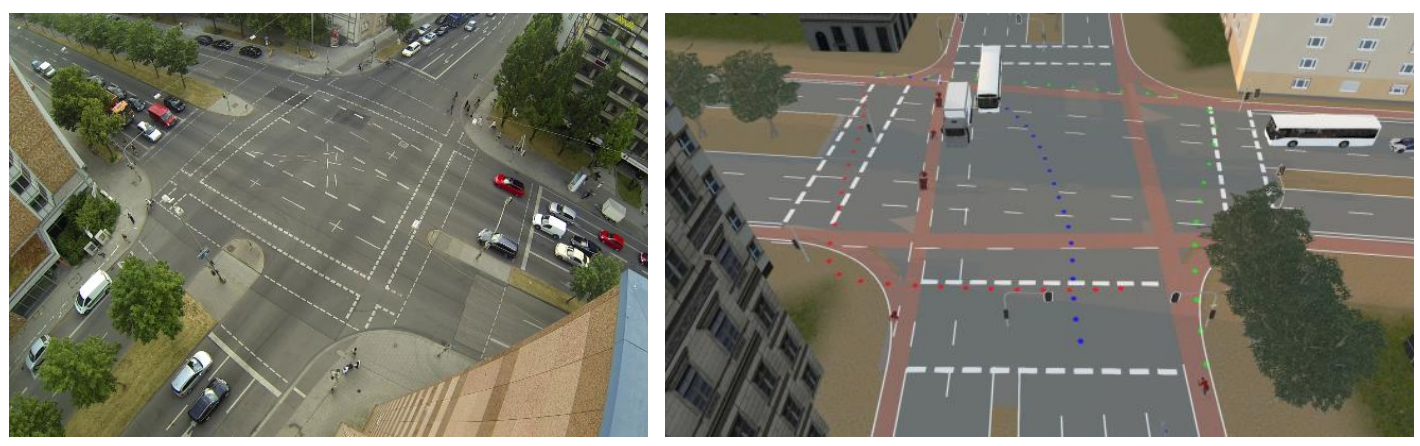

Figure 3: Study intersection Marsstraße/Seidlstraße in Munich, Germany (left: real intersection, right: simulated intersection)

The bicycle simulator at the Chair of Traffic Engineering and Control uses the vehicle and environment simulation DYNA4 to simulate process the raw signals from the hardware setup and to display the environment including surrounding traffic. In order to use the SUMO coupling of the DYNA4 software, the SUMO network is first exported as an OpenDRIVE network using the netconvert SUMO tool. Corrections are made to the OpenDRIVE network using the editor ODDLOT (HLRS HighPerformance Computing Center, 2016). Visual components of the intersection, including buildings and signal heads are added to the simulation in DYNA4. 


\subsection{Guidelines across the intersection}

In Twaddle (2017), trajectories from 1128 bicyclists crossing the Marsstraße/Seidlstraße intersection were extracted from video data using an extended version of the open source software Traffic Intelligence (Saunier, 2016; H. Twaddle, Schendzielorz, Fakler, \& Amini, 2014). The trajectories of bicyclists preforming the same maneuver (e.g., turning left from the south approach to the west exit) were clustered with the purpose of identifying the shape and number of typical pathways used by bicyclists to carry out the maneuver. The exemplar trajectory (or centroid) of each cluster was selected as the average or representative trajectory for the pathway. These exemplars are used in Twaddle (2017) to specify desired pathways across the simulated intersection. Bicyclists in the simulation are assigned a pathway using a tactical behaviour model. Once the pathway is assigned, bicyclists move forward using a proposed operational behaviour model in which bicyclists aim to follow this pathway while responding to other road users and the traffic signal. The cluster trajectories (left) and the exemplars or pathways (right) for bicyclists turning left from the north approach to the east exit of the intersection are shown in Figure 4.
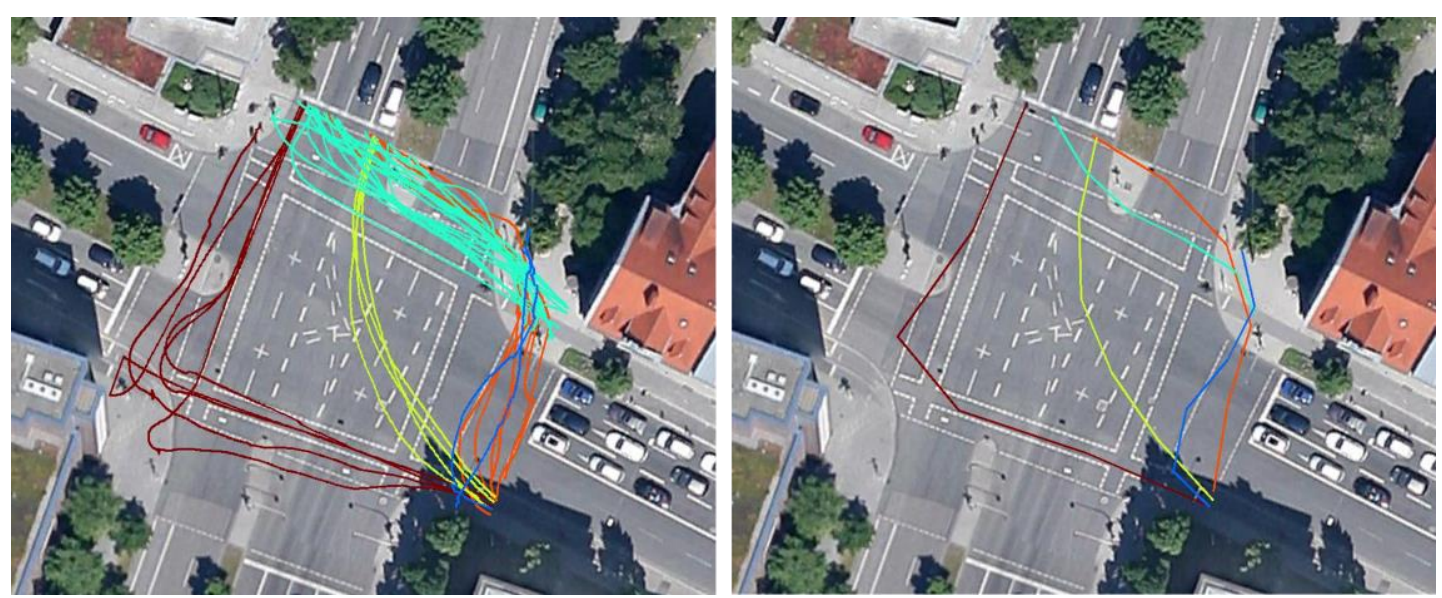

Figure 4: Clustered trajectories from bicy clists turning left from the north approach of the intersection (left) and the cluster exemplars (right) (background images: Google Earth 2013) (Heather Twaddle, 2017)

Here, the cluster exemplars or archetypes of maneuver clusters are used to as a choice set of desired pathways across intersection for the simulator study participants. All exemplars for the desired manoeuvre are displayed in the simulated environment and participants are asked to choose between exemplars by moving along the desired exemplar. These pathways can be represented at the intersection surface above (or below) the lane marking. Once the selected exemplar is identified based on the movement, the other choices are removed from the simulation and the study participant is instructed to follow this pathway as closely as possible while responding normally to other road users, the traffic signal and other obstacles. If neces sary, successive test runs of every available exemplar can be carried out to ensure a large enough sample size of all the pathways to calibrate and validate the behavior models. 


\section{Preliminary Results}

In a first analysis, the trajectories from the study participants in the simulated environment are compared with real trajectories from video observations. Various aspects of operational and tactical bicyclist behavior are assessed:

- Velocity (speed and direction): The velocities realized in the simulated environment are dependent on both the accuracy of the sensors and the ability of the single-track model to recreate bicycle movement. Systematic errors can be detected by comparing velocities, particularly at curves, from bicyclists in real and VR space. Once this calibration of the bicycle simulator is complete, it will be possible to assess the validity of the velocity measurements and potentially use the data to calibrate and validate behavior models.

- Acceleration: This start-up behavior at signalized intersections has been found to be difficult to analyze using the bicycle simulator. Due to the use of a kinematic smart trainer, it is possible for test subject to lean sideways without falling over, which is not the case in reality. This limits the realis $m$ of the acceleration experience for the study participants and may limit the validity of the results. More tests are needed to examine this aspect.

- Interactions with other road users: Interactions are qualified using measures such as Time to Collision (TTC) and Post Encroachment Time (PET), which are sensitive to the accuracy of the velocity measurements. However, the realism of the simulated interacting road users, both in appearance and movement, is also important to ensure interaction data of high quality.

- Path selection: This is a promising aspect of bicyclist behavior to examine with the bicycle simulator. As long as the simulated environment is realistic enough, tactical choice are made in a similar way in the simulated environment and the real world.

\section{Outlook}

The bicycle simulator at TUM VT was developed as a tool for investigating the tactical behavior of bicyclists (e.g., response to a red traffic light). The realism of the simulator experience and the precision of the movement of the bicyclist in the simulated environment are not as critical for this purpose as they are for studies concerning the operational behavior of bicyclists. Validation studies are currently underway to ensure that the behavior of bicycle simulator participants reflects the observed behavior of bicyclists in the real world, both at the tactical and operational behavior levels. The bicycle simulator itself, as well as the models that control the movement of the bicyclist in the simulated environment, are under continual development to improve the realism of the bicycle simulator experience. Once the validity of the bicycle simulator output is verified, trajectories collected in simulator studies can be used for many research purposes.

\section{References}

Amini, S., Twaddle, H., \& Leonhardt, A. (2016). Modeling of the Tactical Path Selection of Bicyclists at Signalized Intersections. In Transportation Research Board 95th Annual Meeting Proceedings.

HLRIS High-Performance Computing Center. (2016). ODDLOT. Retrieved March 1, 2019, from https://www.hlrs .de/solutions -s ervices/service-portfolio/visualization/driving-simulator/oddlot/ 
Kaths, J., Schott, B., \& Chucholowski, F. (2018). Integration des virtuellen Fahrzeugs in die mikroskopische Verkehrsflusssimulation zur virtuellen Erprobung kooperativer Systeme (Integrating the virtual vehicle with microscopic traffic flow simulation for virtual testing of cooperative systems).

Keler, A., Kaths, J., Chucholowski, F., Chucholowski, M., Grigoropoulos, G., Spangler, M., ... Busch, F. (2018). A bicycle simulator for experiencing microscopic traffic flow simulation in urban environments. In 21 st International Conference on Intelligent Transportation Systems (ITSC) (pp. 3020-3023). https://doi.org/10.1109/ITSC.2018.8569576

Saunier, N. (2016). Traffic Intelligence Project. Retrieved February 4, 2016, from https://bitbucket.org/Nicolas/trafficintelligence/wiki/Home

Twaddle, H. (2017). Development of tactical and operational behaviour models for bicyclists based on automated video data analysis. Technische Universität München. Retrieved from http://mediatum.ub.tum.de?id=1366878

Twaddle, H., Schendzielorz, T., Fakler, O., \& Amini, S. (2014). Use of automated video analysis for the evaluation of bicycle movement and interaction. In Proceedings of SPIE - The International Society for Optical Engineering (Vol. 9026). https://doi.org/10.1117/12.2042487 